\title{
Neumonitis por Hipersensibilidad
}

\section{Hypersensitivity pneumonitis}

\author{
Carlos E. Matiz Bueno, MD. ${ }^{1}$
}

\section{Resumen}

La neumonitis por hipersensibilidad corresponde el $20 \%$ de todas las patologías intersticiales; su nueva clasificación en la ATS/ALAT/JRS/ 2020 en inflamatoria o fibrótica y el reconocimiento de los criterios clínicos, patrones radiológicos y patológicos, nos permiten una mayor identificación y mejorar el subdiagnóstico de la enfermedad. La neumonitis depende de una predisposición genética, de la reacción frente a uno o varios antígenos (orgánicos, proteínas e inorgánicos) en forma repetitiva, en una persona susceptible, que lleva a una respuesta inmune y va a desencadenar de acuerdo con el tiempo duración y cantidad de exposición al antígeno una respuesta inflamatoria y posterior fibrótica en la vía aérea pequeña y el parénquima pulmonar. Su incidencia y prevalencia son variables de acuerdo con la región y la identificación del antígeno. El cuadro clínico dependerá de la exposición aguda o crónica al antígeno, aunque algunos pacientes pueden desarrollar la forma crónica a pesar de la suspensión del antígeno. El diagnóstico se realiza con una historia clínica completa, detallada, tratando de identificar el antígeno, el cuadro clínico y los patrones radiológicos y patológicos inflamatorios o fibróticos, asociado a linfocitosis del $20 \%$ en el lavado broncoalveolar y la ayuda de un grupo multidisciplinario. La suspensión de la exposición al antígeno es lo más importante del tratamiento; la terapia farmacológica se realiza con inmunosupresores, esteroides, micofenolato y azatioprina con baja evidencia y los anti fibróticos están indicados en la forma fibrosante progresiva.

Palabras Clave: neumonitis por hipersensibilidad; enfermedad pulmonar intersticial difusa; Asociación Latinoamericana de Tórax; Tomografía Axial Computarizada de Tórax; Neumonía intersticial usual; Fibrosis pulmonar Idiopática, neumonía intersticial no específica.
${ }^{1}$ Internista Neumólogo Fundación Santa Fe de Bogotá. Profesor de Medicina, Universidad de los Andes

\author{
Autor de Correspondencia \\ Carlos E. Matiz. \\ Correo electrónico: \\ carlosmatiz@hotmail.com
}

Recibido: 10/08/2021

Aceptado: 29/11/2021 


\begin{abstract}
Hypersensitivity pneumonitis corresponds to $20 \%$ of all interstitial pathologies, its new classification of the ATS / ALAT / JRS / 2020 in inflammatory or fibrotic and identification of clinical criteria, radiological and pathological patterns allow us a greater identification and improve the subdiagnosis of the illness. Pneumonitis depends on a genetic predisposition, on the reaction against one or more antigens (organic, protein and inorganic) repeatedly, in a susceptible person, which leads to an immune response and will trigger according to time, duration and quantity of antigen exposure an inflammatory and subsequent fibrotic response in the small airway and lung parenchyma. Its incidence and prevalence are variable according to the region and the identification of the antigen. The clinical picture will depend on the acute or chronic exposure to the antigen, although some patients may develop the chronic form despite the suspension of the antigen. The diagnosis will be made with a complete, detailed clinical history, seeking, trying to find the antigen, the clinical picture and the inflammatory or fibrotic radiological and pathological patterns, associated with $20 \%$ lymphocytosis in the bronchoalveolar lavage and the help of a multidisciplinary group. Suspension of antigen exposure is the most important part of treatment. Pharmacological therapy is performed with immunosuppressants, steroids, mycophenolate, and azathioprine with low evidence, and antifibrotic drugs are indicated in the progressive fibrosing form.
\end{abstract}

Keywords: Hypersensitivity pneumonitis, Diffuse interstitial lung disease, Latin American thorax association, Computed tomography of the chest, Usual interstitial pneumonia, Idiopathic pulmonary fibrosis, Non-specific interstitial pneumonia, American thoracic society, Japanese respiratory society, American college of chest physicians.

\section{Introducción}

La neumonitis por hipersensibilidad $(\mathrm{NH})$ es una enfermedad inflamatoria o fibrótica que afecta el parénquima pulmonar y la vía aérea pequeña. Es el resultado de una reacción inmune provocada por un antígeno inhalado en individuos susceptibles. La palabra susceptible es muy importante ya que todas las personas expuestas a un antígeno no desarrollan la enfermedad. En términos generales, el desarrollo de $\mathrm{NH}$ depende del tiempo de exposición, el tipo de antígeno o antígenos y la susceptibilidad genética $(1,2)$. La NH se manifiesta como una enfermedad pulmonar intersticial y debe considerarse siempre en el diagnóstico diferencial en una nueva enfermedad pulmonar intersticial. Lo más importante es la sospecha en el interrogatorio y la historia clínica de un antígeno, sea orgánico o no orgánico que la va a diferenciar de las otras patologías intersticiales.

\section{Clasificación}

Las clasificaciones de la neumonitis de hipersensibilidad han cambiado a través del tiempo a medida que se ha avanzado en el conocimiento de la enfermedad y su abordaje multidisciplinario con clínicos, radiólogos y patólogos, lo que ha permitido reconocer mecanismos fisiopatológicos, patrones radiológicos e histopatológicos para hacer el diagnóstico definitivo de la enfermedad. Desde 1989 se clasificó como una enfermedad aguda, subaguda y crónica (3).

En 2017 se cambió la clasificación basada en la duración de los síntomas y hallazgos radiológicos y patológicos, en aguda (menos de 6 meses) y crónica (más de 6 meses) (4). En el 2020 se realiza el Consenso de la ATS, JRS, ALAT en la cual se consolida el conocimiento en lo clínico, en los patrones tomográficos y patológicos y se determinó por consenso clasificar la neumonitis de hipersensibilidad por su presentación clínica, radiológica y patológica como fibrótica (mezcla de inflamación y fibrosis o puramente fibrótica) o no fibrótica (puramente inflamatoria).

Algunos expertos la determinan como criptogénica o NH sin causa determinada, cuando no se encuentra antígeno (2).

\section{Epidemiología}

La incidencia de la enfermedad es variable y no se conoce con precisión, principalmente por un subdiagnóstico. En la NH no fibrótica, porque no se identifica fácilmente, no se registra y se confunde con otras patologías especialmente infecciosas y en la $\mathrm{NH}$ fibrótica por la dificultad en el diagnóstico diferencial 
con diferentes neumopatías intersticiales como, por ejemplo, la neumonía intersticial no específica y la fibrosis pulmonar idiopática. De todas las patologías intersticiales la neumonitis por hipersensibilidad representa un $20 \%$ aproximadamente $(5,6)$. En Nuevo México la incidencia de $\mathrm{NH}$ entre las enfermedades intersticiales es de $30 \%$, en Canadá se encontró en el $30 \%$ de 661 pacientes, en España en el $18 \%$ de 500 pacientes, en India en $47 \%$ de 513 pacientes y en Dinamarca 4.1 por 100.000 /año (6). Esa variabilidad también depende del antígeno inductor documentado en forma local o regional en los diferentes países, en lo cual debemos insistir para mejorar la epidemiologia de la enfermedad, ya que depende del clima, ocupación, hobbies y exposiciones ambientales. En los niños la enfermedad es muy poco común con una prevalencia de 4 por 100.000 (7).

\section{Clínica y curso de la enfermedad}

La presentación clínica de la neumonitis por hipersensibilidad depende del tipo del antígeno, de la duración de la exposición (continua o intermitente) o que exista uno o más antígenos inhalados. Se presenta en adultos jóvenes y mayores de edad y en estos últimos (>65 años) predomina el patrón fibrótico. La forma aguda inflamatoria no fibrótica se caracteriza por tos seca, disnea, fiebre, mialgias y puede presentarse entre 6 a 8 horas después de una exposición masiva al antígeno; puede simular un resfrío o patología infecciosa y los síntomas pueden desaparecer en las primeras 24 horas o en pocos días (1).

La forma crónica o fibrótica se caracteriza por disnea, tos, estertores al final de la inspiración y pérdida de peso según la cronicidad de los síntomas. Según la duración de la exposición al antígeno(s) se puede presentar disnea progresiva, con deterioro de su clase funcional, empeoramiento de la fibrosis en la tomografía computada de alta resolución (TACAR) y falla respiratoria (8).

En algunos casos puede existir una exacerbación de la enfermedad con empeoramiento de la disnea $\mathrm{e}$ incremento de los infiltrados en TACAR, con aumento de las imágenes de vidrio esmerilado. Aquí es necesario descartar una patología infecciosa, falla cardiaca o tromboembolismo pulmonar.

El curso y pronóstico es favorable al retirarse de la exposición con remisión de los síntomas, pero si se continúa con exposición al antígeno se desarrolla la forma fibrótica. Existen condiciones de peor pronóstico como el ser hombre mayor, fumador, con capacidad vital forzada (CVF) de inicio disminuida, que no se encuentre el antígeno, ausencia de linfocitosis en el lavado bronquioalveolar (BAL) y que tenga un patrón tipo neumonía intersticial usual (NIU) en la TACAR (9). La diversidad en el cuadro clínico se correlaciona con el patrón radiológico e histopatológico. Teóricamente la secuencia es: un cuadro inicial inflamatorio, un cuadro mixto inflamatorio y fibrótico, uno puramente fibrótico, un cuadro progresivo fibroproliferativo y finalmente un cuadro terminal respiratorio (10). En el seguimiento de estos pacientes, es fundamental la correlación clínica y funcional de la espirometría y curva flujo volumen y la difusión de monóxido de carbono cada 3 a 6 meses. Los hallazgos de progresión tomográfica de la enfermedad incluyen la presencia de bronquiectasias por tracción, panalización y o patrón de NIU. Los hallazgos de progresión funcional incluyen un descenso de la CVF $>10 \%$ o difusión de CO de $>15 \%(11-24)$.

\section{Patogénesis}

\section{Antígenos}

Existen aproximadamente 200 antígenos o agentes inductores que pueden causar la neumonitis por hipersensibilidad. El antígeno varía dependiendo de la geografía, a nivel local o regional, pueden ser orgánicos (los más frecuentes) e inorgánicos y varían de acuerdo con su peso molecular. Los de más bajo peso molecular son agentes químicos, por ejemplo, el diisocianato de tolueno. Los orgánicos son derivados de proteínas de diversos microorganismos incluyendo bacterias termofílicas, hongos, micobacterias, o de animales (principalmente aves) (4-8) (Tabla 1).

En el caso de la exposición relacionada con el pulmón del granjero o agricultor y de los que trabajan en la industria pueden existir múltiples antígenos. La identificación o sospecha del antígeno es fundamental

Tabla 1. Agentes que causan neumonitis de hipersensibilidad (2)

\begin{tabular}{|lll|}
\hline \multicolumn{1}{|c}{ Orgánicos } & \multicolumn{1}{c|}{ Proteínas/Enzimas } & \multicolumn{1}{c|}{ Inorgánicos } \\
1. Microbios & 1. Animal & 1. Químicos \\
2. Hóngos & 2. Vegetal & 2. Farmacéuticos \\
3. Levaduras & & 3. Metales \\
4. Hongo comestible & \\
5. Bacteria & & \\
6. Protozoos & & \\
\hline
\end{tabular}


para diferenciar la NH de otras enfermedades pulmonares intersticiales. La búsqueda del agente con una historia clínica completa, un interrogatorio exhaustivo de la ocupación, hobbies, la vivienda, entre otros aspectos, permitirá determinar el agente causal. Desafortunadamente el antígeno responsable se identifica solamente en un $40 \%$ a $50 \%$ de los casos (12).

En algunos casos puede ocurrir una exposición intermitente $o$ en otros, la inhalación de un segundo antígeno puede llevar a la forma progresiva fibroproliferativa. Con respecto a la recomendación de la guía ATS/ALAT/JRS de los cuestionarios específicos para la $\mathrm{NH}$ fibrótica o no fibrótica y detección del antígeno, no existe recomendación o sugerencia en pro o en contra de la realización de cuestionarios específicos o universales para identificar el agente potencial, por lo que se sugiere realizar cuestionarios propios de cada región, en los que se incluyan condiciones ambientales y ocupacionales relevantes a la geografía y hábitos culturales de cada lugar (2).

Con respecto a la pregunta del consenso de la guía del CHEST de expertos 2021: ¿se debe solicitar en pacientes con sospecha de $\mathrm{NH}$, únicamente en el suero el antígeno especifico de la inmunoglobulina IgG o inmunoglobulina A IgA para confirmar o descartar la NH? La recomendación es débil, muy bajo nivel de evidencia. Esta respuesta es atribuible a la mala estandarización de la prueba, la baja estandarización del antígeno $\operatorname{IgG} / \operatorname{IgA}$, la baja técnica de inmunoensayo y puntos de corte de la cuantificación de la IgG (27).

Otra pregunta del mismo consenso de expertos del CHEST 2021 es respecto a la utilización de la prueba de reto de exposición al antígeno como método de diagnóstico de la NH. La recomendación es baja, con bajo nivel de evidencia. La realización de la prueba de reto para el diagnóstico de la $\mathrm{NH}$ también tiene limitación en la estandarización, cantidad del antígeno expuesto, sitio de la prueba y la validación cuando la respuesta es positiva. El hecho de ser negativa no significa que la persona no estuvo expuesta a ese antígeno y no descarta la causa de la NH $(1,27)$.

La guía no califica, pero considera importante incluir un médico de salud ocupacional, cuando la búsqueda del antígeno sea difícil o no este clara (27).

\section{Genética}

La susceptibilidad genética es un factor importante en el desarrollo. Los polimorfismos en genes del Complejo Principal de Histocompatibilidad clase II (MHC), (HDLA-DR3,DR7,DQ3,DRB1 $\left.{ }^{\star} 04\right)$, en genes del proteasoma, de proteínas trasportadoras e inhibidores de las metaloproteinasas, han sido asociados con esta enfermedad $(2,10,13,14)$. Se ha documentado, al igual que en la fibrosis pulmonar idiopática, una asociación con una pobre sobrevida de un polimorfismo de la mucina (MUC5B) y de genes relacionados con la biología de los telómeros. Las mutaciones en estos últimos provocan un acortamiento grave de los telómeros y se han descrito tanto en neumonitis de hipersensibilidad familiar como esporádica (14) (Ver Tabla 2).

\section{Respuesta inmune}

La respuesta inmune después de la exposición al antígeno empieza con su fagocitosis por macrófagos, su procesamiento por las vías MHC I y II y su presentación a linfocitos $\mathrm{T}$ y $\mathrm{B}$, lo que resulta en una respuesta humoral (mediada por anticuerpos IgG) y celular por linfocitos $\mathrm{T}$ ayudadores (Th1), son las células predominantes en la enfermedad, con liberación de citocinas como el IFN-y, resultando en el cuadro clínico característico no fibrótico o inflamatorio, con la formación de granulomas en la histopatología y la bronquitis, bronquiolitis y neumonitis linfocítica.

Si la exposición al antígeno continua o existe un segundo antígeno o una respuesta inmune repetida, se puede desarrollar la parte fibroproliferativa de la enfermedad (fibrótico), con secreción de citocinas profibróticas como TGF-ß, IL-4, e IL-13 con acumulación de fibroblastos, depósito exagerado de proteínas de matriz extracelular y la apoptosis 
Tabla 2. Factores genéticos en neumonitis por hipersensibilidad

\begin{tabular}{|c|c|c|}
\hline Variantes genéticas & Etnia & Autor referencia \\
\hline \multicolumn{3}{|l|}{ MH II Polimorfismos } \\
\hline HLA-DR3 & Blanco & Ritner 1986 \\
\hline HLA-DR7 & Mexicano & Selman 1987 \\
\hline HLA-DQ3 & Japonés & Ando 1989 \\
\hline HLA-DRB1'04 & Mexicano & Farfán 2014 \\
\hline MHCII Haplotipos & Mexicano & Camarena 2001 \\
\hline \multicolumn{3}{|l|}{ Aumento DRB1'1305 } \\
\hline \multicolumn{3}{|l|}{ Disminución DRB1 0802} \\
\hline \multicolumn{3}{|l|}{ Proteasoma y trasportados } \\
\hline \multicolumn{3}{|l|}{ Polimosfismos } \\
\hline PSMB8 KQ & Mexicano & Camarea 2010 \\
\hline TAPI 637,661 & Mexicano & Aquino - Gálvez \\
\hline \multicolumn{3}{|l|}{ Polimorfismos mucina } \\
\hline MUC5B rs 35705950 & Blanco & Ley 217 \\
\hline \multicolumn{3}{|l|}{ Mutaciones del telomero } \\
\hline Acortamiento de telomero & Blanco & Ley 217 \\
\hline Mutación telomero & Blanco & Newton 2016 \\
\hline Polimorfismo antiproteasa & Blanco & Hill 2014 \\
\hline
\end{tabular}

epitelial característica de NH fibrótica (10-15).

\section{Hallazgos radiológicos}

La identificación de los patrones radiológicos tomográficos es uno de los avances de la enfermedad. El consenso ATS/JRS/ALAT 2020 y CHEST 2021 permitieron unificar los criterios radiológicos en un patrón típico, compatible e indeterminado de la neumonitis por hipersensibilidad similar a lo logrado con la FPI. Estos patrones se correlacionan con los hallazgos histopatológicos no fibróticos (inflamatorios) y fibróticos. Es fundamental que se realicen los cortes de la tomografía en inspiración y espiración, se busque el patrón de mosaico en las 
imágenes en inspiración y el atrapamiento aéreo en la espiración, los cuales son hallazgos semiológicos que ayudan a confirmar el diagnóstico de neumonitis por hipersensibilidad. Finalmente, la identificación de la combinación de las tres densidades en inspiración: de pulmón normal, vidrio esmerilado y pulmón hiperlucente, ayuda a confirmar el diagnóstico (2-17). Los patrones indeterminados (neumonía intersticial usual, no especifica en la fase fibrótica y neumonía en organización) tienen que ser llevados a biopsia por broncoscopia, criobiopsia o toracoscopia según la disponibilidad y experiencia del centro y tener un seguimiento por grupo multidisciplinario.

En las Tablas 3 y 4 se muestran las características de los patrones tomográficos diagnósticos típicos, compatible, o indeterminado de la $\mathrm{NH}$ no fibrótica y fibrótica.

Tabla 3. Tomografía de tórax patrón no fribrótico de neumonitis de hipersensibilidad (2)

\begin{tabular}{|c|c|c|c|}
\hline Patrón & Típico & Compatible & Indeterminado \\
\hline Descripción & $\begin{array}{l}\text { 2. Un hallazgo TACAR indicativo de enfermedad } \\
\text { vía aérea pequeña en una distribución difusa }\end{array}$ & & \\
\hline \multirow[t]{6}{*}{$\begin{array}{l}\text { Hallazgos } \\
\text { radiológicos } \\
\text { relevantes }\end{array}$} & $\begin{array}{l}\text { TACAR anormalidades con infiltración } \\
\text { parenquimatosa }\end{array}$ & Anormalidades parenquimatosas & NA \\
\hline & 1. Vidrio esmerilado & 1. Vidrio esmerilado uniforme & \\
\hline & 1. Nódulos centilobulares mal definidos & 1. Craniocaudal difusa (predominio basal) & \\
\hline & 2. Atrapamiento aéreo & $\begin{array}{l}\text { 2. Axial: difusa (variante peribronco } \\
\text { vascular) }\end{array}$ & \\
\hline & Distribución parenquimatosa & & \\
\hline & $\begin{array}{l}\text { 1. Craniocaudal difusa (con o sin compromiso } \\
\text { basal) }\end{array}$ & & \\
\hline
\end{tabular}


Tabla 4. Tomografía de tórax patrón fibrótico de neumonitis de hipersensibilidad (2)

\begin{tabular}{|c|c|c|c|}
\hline Patrón & Típico & Compatible & Indeterminado \\
\hline \multirow[t]{4}{*}{$\begin{array}{l}\text { Hallazgos } \\
\text { radiológicos } \\
\text { relevantes }\end{array}$} & $\begin{array}{l}\text { Anormalidades del TACAR con fibrosis pulmonar } \\
\text { compuesto por opacidades linear, reticulación, con } \\
\text { distorsión pulmunar, bronqui Ectasias y panalización } \\
\text { presente pero no predomina. }\end{array}$ & $\begin{array}{l}\text { Variante patrón de fibrosis: } \\
\text { 1. Patrón de FPI diagnóstico por guías } \\
\text { del } 2018 \\
\text { 2. Extenso vidrio esmerilado con áreas de } \\
\text { fibrosis }\end{array}$ & $\begin{array}{l}\text { Patrones solos: } \\
\text { 1. Patrones de NIU } \\
\text { 2. Probable patrón de NIU } \\
\text { 3. Patrón de indeterminado de } \\
\text { NIU } \\
\text { 4. NINE fibrosante } \\
\text { 5. Neumonía en organización } \\
\text { 6. Patrón indeterminado }\end{array}$ \\
\hline & TACAR con compromiso de vías aéreas pequeñas: & $\begin{array}{l}\text { TACAR con compromiso vía aérea } \\
\text { pequeña: }\end{array}$ & \\
\hline & $\begin{array}{l}\text { 1. Nódulos mal definidos centrilo bular y/o vidrio } \\
\text { esmerilado }\end{array}$ & 1. Nódulo cetrilobular mal definido & \\
\hline & $\begin{array}{l}\text { 2. Mosaico atenuación, patrón de tres densidades y/o } \\
\text { atrapamiento aéreo }\end{array}$ & $\begin{array}{l}\text { 2. Patrón de tres densidades y/o } \\
\text { atrapamiento aéreo }\end{array}$ & \\
\hline
\end{tabular}

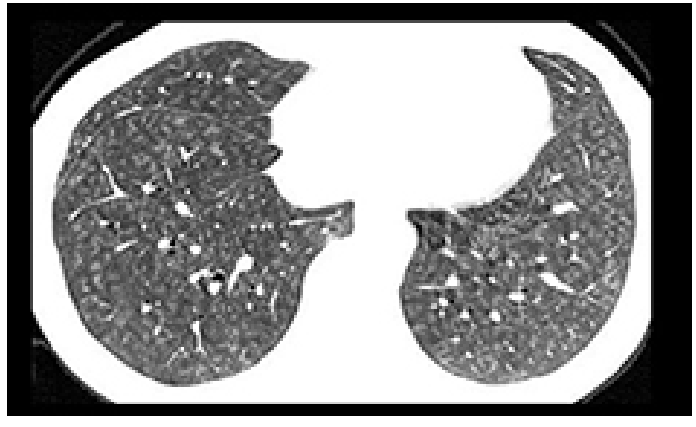

Figura 1. Patrón no fibrótico con nódulos en formación y vidrio esmerilado en neumonitis por hipersensibilidad no fibrótica.

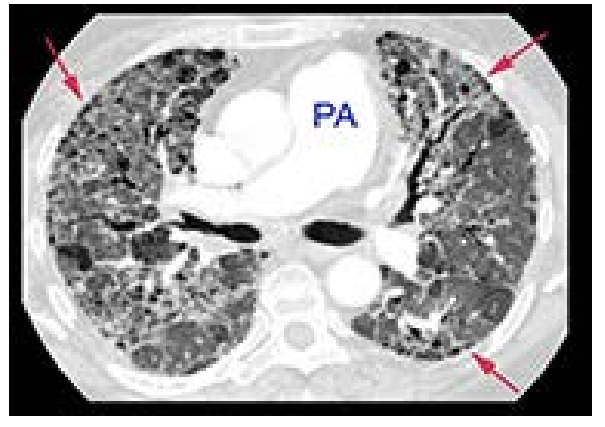

Figura 2. Patrón fibrótico de la neumonitis por hipersensibilidad con compromiso apical con vidrio esmerilado, bronquiectasias de tracción, mosaico de atenuación, las tres densidades y áreas de panalización. 


\section{Patrones histopatológicos}

Los patrones histopatológicos al igual que los patrones radiológicos según el consenso ATS/ JRS/ALAT 2020 permitieron resumir los criterios encontrados en la literatura hasta la fecha y el análisis de los expertos para clasificar los patrones como: definitivo, probable e indeterminado de esta enfermedad. Después de realizar la historia clínica completa, la búsqueda del antígeno, la presencia o ausencia de linfocitosis en el lavado bronquioloalveolar y un TAC de tórax con un patrón probable o indeterminado, si no se tiene el diagnóstico, se debe llevar a un análisis del grupo multidisciplinario para decidir la realización de la biopsia para confirmar el diagnóstico y decidir el tratamiento (2-9-16).

En la Figura 3 se muestran los hallazgos histopatológicos de la neumonitis por hipersensibilidad definitivo, probable e indeterminado en la clasificación no fibrótica.

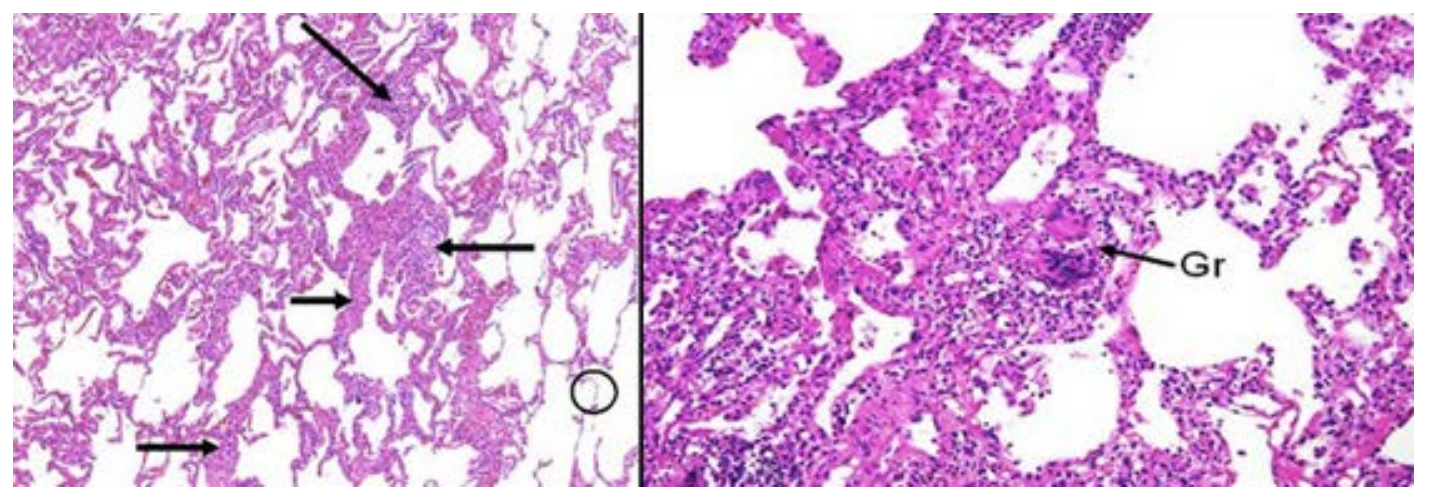

Figura 3. Patrón histopatológico de neumonitis por hipersensibilidad con patrón homogéneo de neumonía intersticial no específica, con formación de granulomas no necrotizantes, con puentes, predominio de linfocitos y compromiso bronquiolocéntrico. 
Tabla 5. Clasificación histopatológia no fibrótica de la NH(2)

\begin{tabular}{|c|c|c|}
\hline NH & Probable & Indeterminado \\
\hline $\begin{array}{l}\text { Nofibrotico NH (Celular) } \\
\text { Cambios histopatológicos típicos no fibroticos al } \\
\text { menos una biopsia mostrando tres hallazgos: } \\
\text { 1. Neumonía intersticial celular } \\
\text { - NINE celular } \\
\text { - Predominio linfocítico } \\
\text { 2. Broquiolitis celular } \\
\text { - Predominio linfocítico; nomas de foco linfoide } \\
\text { peribronquiolar con centros germinales } \\
\text { - Neumonía en organización con cuerpos de } \\
\text { Masson } \\
\text { - Macrófagos en espacio aéreo terminal } \\
\text { 3. Granulomas pobremente formados no } \\
\text { necrotizante } \\
\text { - Clúster de cedular epitelioides, multinucleada con } \\
\text { inclusiones intracitoplasmáticas } \\
\text { - Localizada en intersticio peribroquiolar y/o } \\
\text { neumoníaen organización } \\
\text { - Ausencia células plasmáticas, células } \\
\text { plasmáticas>linfocitos } \\
\text { - Hiperplasia linfoide, granuloma sacroidal o } \\
\text { necrotizante y partículas aspiradas }\end{array}$ & $\begin{array}{l}\text { 1-2 criterios de primera columna (NH) en una } \\
\text { biopsia } \\
\text { 1. Neumonía intersticial bronquiolocentrico } \\
\text { - NINE celular } \\
\text { - Predominio linfocítico } \\
\text { 2. Bronquiolitis celular } \\
\text { - Predominio celular } \\
\text { - Neumonía en organización } \\
\text { - Macrofágos en vía aérea terminal } \\
\text { - Ausencia células plasmáticas, células } \\
\text { plasmáticas>linfocitos } \\
\text { - Hiperplasia linfoide, granuloma sacroidal o } \\
\text { necrotizante y partículas aspiradas }\end{array}$ & $\begin{array}{l}\text { Una biopsia demostrando 1-2 criterios de la } \\
\text { primera columna (NH) } \\
\text { Patrones seleccionados } \\
\text { - NINE celular } \\
\text { - Neumonía en organización } \\
\text { - Metaplasia peribronquiolar } \\
\text { - Ausencia células plasmáticas, células } \\
\text { plasmáticas> linfocitos } \\
\text { - Hiperplasia linfoide, granuloma sacroidal o } \\
\text { necrotizante y partículas aspiradas }\end{array}$ \\
\hline
\end{tabular}

Tabla 6. Clasificación histopatológica patrón fibrótico de NH (2)

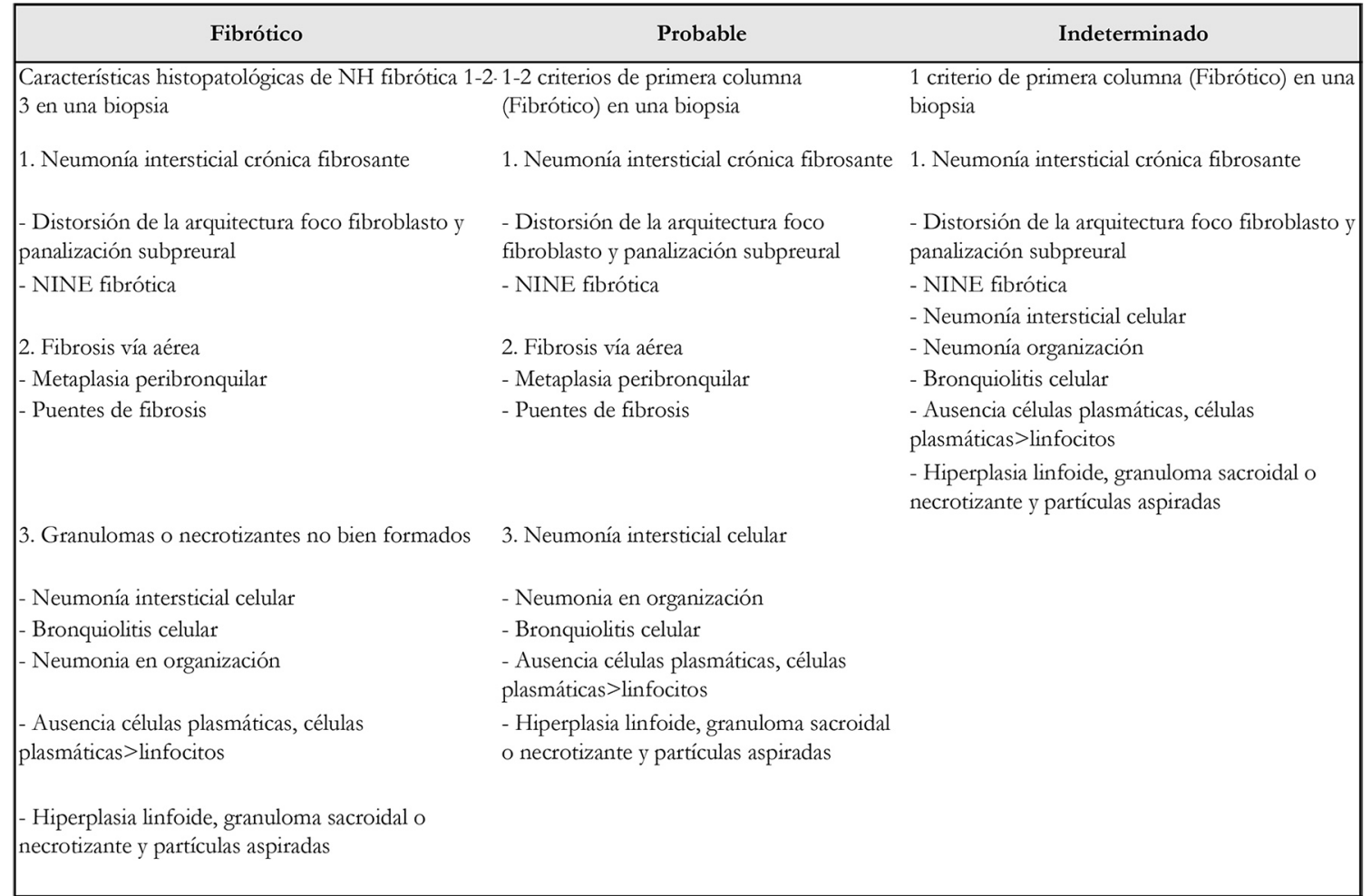




\section{Diagnóstico}

El diagnóstico de la neumonitis por hipersensibilidad continúa siendo un reto para el médico. Esto es importante porque es de las pocas enfermedades intersticiales que tiene causa y que debemos insistir en la búsqueda del antígeno causal, a través de una historia clínica detallada, investigando no solo los antígenos más frecuentes como aves, moho, exposición en las fincas o trabajos de agricultura (12-19), sino en la ocupación en forma detallada, hobbies, vivienda, cambios de hábitat, ambiente y medicamentos utilizados por el paciente. Se debe identificar si los síntomas son agudos o crónicos y si el paciente es mayor o menor de 50 años, para clasificarlo como no fibrótico o fibrótico. El paciente no fibrótico puede presentar síntomas agudos o de pocas semanas/meses de evolución, es más frecuente identificar la fuente de exposición y encontrar nódulos en formación en la tomografía y linfocitosis en el BAL (2).

El paciente fibrótico presentará síntomas más insidiosos, crónicos, con una mayor dificultad en el hallazgo del antígeno que sólo se encuentra en alrededor de $40 \%$ a $50 \%$. En la TACAR presenta patrón fibrótico y en el BAL linfocitosis leve o moderada (2).

Por este motivo, la guía de ATS/JRS/ALAT enfatiza en tres dominios para el diagnóstico:

1. Identificación en la exposición (historia clínica con o sin cuestionario, medición de IgG contra el antígeno o test de especifico de inhalación).

2. Patrón tomográfico.

3. Linfocitosis en el BAL o hallazgos histopatológicos compatibles.

La NH puede ser diagnosticada con alto índice de confianza en pacientes con identificación del antígeno, una TACAR de tórax con patrón definitivo y linfocitosis en el BAL. Este paciente no requiere ningún otro examen adicional según grupo multidisciplinario (18). Los pacientes que tengan otra combinación de historia clínica, de hallazgo o no del antígeno, del patrón compatible o indeterminado de la tomografía y presencia o ausencia de linfocitosis en el BAL, requieren de la evaluación del grupo multidisciplinario (clínico, radiólogo, patólogo, reumatólogo) para definir la necesidad de la biopsia ya sea por criobiopsia o por toracoscopia $(2-18)$ (Figura 4).

Las preguntas PICO realizadas por el consenso ATS/JRS/ALAT permitieron realizar una revisión sistemática de la literatura y aclarar las dudas sobre los métodos diagnósticos y su recomendación para consolidar el conocimiento de la enfermedad.

1. Con respecto a si debemos realizar la medición de test serológico para anticuerpos IgG contra antígenos específicos, el consenso ATS/ JRS/ALAT 2020- concluyó que debido a su variabilidad en la sensibilidad $83 \%-90 \%$ y especificidad $68 \%-91 \%$ los resultados son subóptimos, insuficientes para confirmar o excluir el diagnóstico de NH. Es importante destacar que una prueba de $\operatorname{IgG}$ positiva no significa que el antígeno sea la causa, sino que solamente estuvo expuesto en algún momento de su vida (2). Sin embargo, su recomendación sugiere realizar el estudio para potenciales antígenos que causen $\mathrm{NH}$.

2. ¿Se debe realizar un conteo de linfocitos en el BAL para el diagnóstico de neumonitis de hipersensibilidad? El consenso concluye que después de una revisión sistemática recomienda fuertemente este estudio para HP no fibrótica y con menor confianza para HP fibrótica (2-1220).

3. ¿Se debe indicar la biopsia transbronquial para el diagnóstico de NH?

Recomendación: No fibrótica, sugiere la biopsia transbronquial (muy baja confianza estimada); en la fibrótica no realiza recomendación a favor o en contra de la biopsia transbronquial (2-23).

4. ¿Se debe realizar una criobiopsia para diagnóstico de NH? Recomendación: No fibrótica, no recomendación o sugerencia a favor o en contra de criobiopsia; en NH fibrótica, sugiere la criobiopsia (2-21-22).

5. ¿Se debe realizar una biopsia quirúrgica para diagnóstico de neumonitis por hipersensibilidad? Recomendación: se sugiere biopsia quirúrgica tanto en no fibrótico como fibrótico, cuando se hayan agotado las otras alternativas diagnósticas (2).

\section{Tratamiento}

El tratamiento más importante y definitivo es, rápidamente retirarse de la fuente de exposición que esté causando la enfermedad sea en el trabajo, en la casa, en la realización de un pasatiempo o en el medio ambiente que pueda ser sospechoso. Inclusive se pueden tomar fotos o videos del entorno cuando no hay un agente claro o un segundo agente que puede estar perpetuando su enfermedad, para ser examinado por el médico y/o un experto ocupacional 

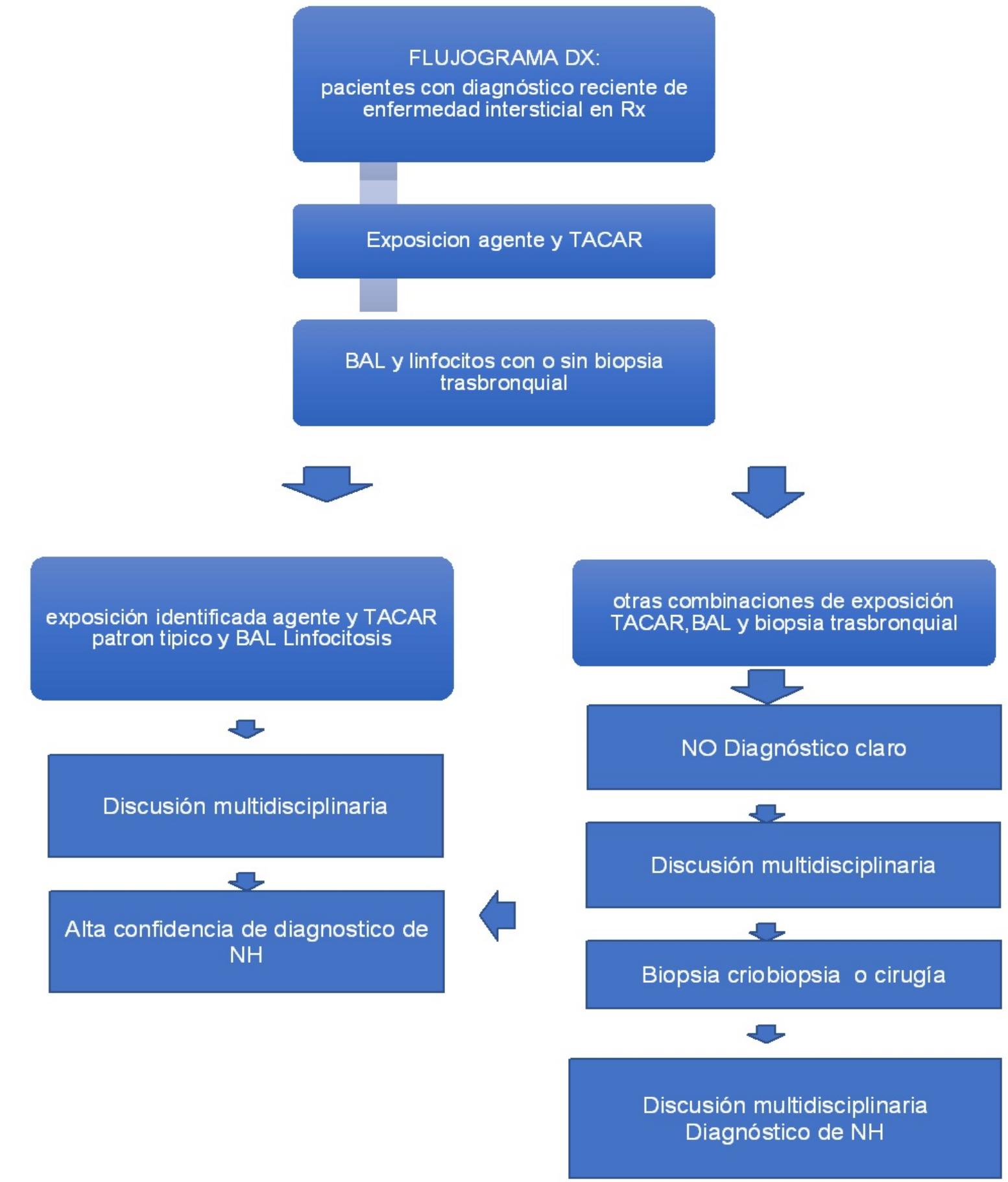

Figura 4. Flujograma diagnóstico de la neumonitis por hipersensibilidad. 
y para poder tomar conductas de manejo. El retiro del antígeno y la mejoría clínica dependerán del antígeno o varios antígenos expuestos, la duración y la intensidad del tratamiento de elección en la forma no fibrótica o fibrótica. La identificación del antígeno será de mejor pronóstico que no encontrarlo, pero en la forma fibrótica el hecho de retirar el antígeno no significa que no puede progresar la neumonitis por hipersensibilidad crónica. Debemos trabajar con los médicos de salud ocupacional en caso de que sea necesario, frente a todas las medidas de prevención, máscaras, retiro o cambio de material que tengan que ver con la etiología de la $\mathrm{NH}(1,27)$.

Con relación al tratamiento farmacológico, existen escasos estudios que sean doble ciego, aleatorizados y controlados. Los corticoesteroides son el tratamiento más utilizado sin estudios que lo confirmen con respecto a la dosis (prednisona $0.5 \mathrm{mg} / \mathrm{kg}$ ), duración y efectividad. Este fármaco es más útil en la fase no fibrótica que la fibrótica de la enfermedad. La forma no fibrótica responderá con el retiro del antígeno únicamente y ocasionalmente podemos dar tres meses de esteroides orales y o inmunomoduladores. Estos resultados están basados en estudios observacionales con bajo número de pacientes $(1,10,19)$.

La terapia inmunomoduladora se ha utilizado sin evidencia científica en pacientes con $\mathrm{NH}$ fibrótica, especialmente en pacientes que no han mejorado con el retiro de la exposición y el uso de corticoesteroides. Se han realizado estudios con bajo número de pacientes y no aleatorizados con azatioprina $o$ micofenolato agregado a bajas dosis de prednisona. En un estudio retrospectivo que incluyó 50 pacientes con $\mathrm{NH}$ fibrótica, con el uso de azatioprina o micofenolato presentaron mejoría en la difusión de $4.2 \%$ año, pero sin cambios en la CVF (25).

Otros estudios han sido realizados con rituximab o leflunomide sin evidencia científica clara de posible utilidad y con bajo número de pacientes $(1,10,19)$.

En la fase fibrótica debe usarse rehabilitación respiratoria, uso de oxígeno en pacientes con hipoxemia e hipertensión pulmonar y trasplante pulmonar, como otra forma de manejo que mejora la sobrevida a cinco años, comparada con la FPI (4-19).

Un estudio prospectivo, aleatorizado, doble ciego controlado con placebo de 52 semanas (INBUILD), evaluó la eficacia y seguridad de nintedanib en 663 pacientes mayores de 18 años con fibrosis pulmonar progresiva, 84 de ellos $(23 \%)$ con NH. Todos los pacientes tenían compromiso fibrosante $>10 \%$ en TACAR, con promedio de CVF $68 \%$ y DLCO $44.4 \%$ del predicho y con $62 \%$ con patrón tomográfico de NIU. El objetivo primario era la disminución anual de la CVF. Los resultados mostraron una disminución de la caída de la CVF de toda la población en estudio de $107 \mathrm{ml} /$ año frente a placebo. En los pacientes que tenían patrón tomográfico de NIU, la disminución de la CVF de los que recibían nintedanib fue de 82.9 $\mathrm{ml}$ por año, frente a $211 \mathrm{ml}$ que recibían placebo, con una diferencia de $128.2 \mathrm{ml}$, mostrando un beneficio en el grupo que recibía nintedanib en los pacientes con fibrosis pulmonar progresiva (26). Es muy importante el seguimiento funcional de este tipo de pacientes con NH y Fibrosis Pulmonar Progresiva durante dos años o antes, si existe un deterioro clínico, funcional y tomográfico. Se debe evaluar la caída del $10 \%$ de la CVF y $15 \%$ de la difusión de monóxido de carbono (CO) y seguimiento clínico y radiológico (tomográfico) de la extensión más del $20 \%$, presencia de bronquiectasias de tracción, incremento de vidrio esmerilado y o panalización, con patrón de NIU. También puede documentarse una caída del 5 a $10 \%$ de las pruebas funcionales asociadas a cambios clínicos y tomográficos, como signos de progresión de la enfermedad (11).

Un estudio de Pirfenidona (RELIEF) en pacientes con fibrosis pulmonar progresiva, en el cual existía un $45 \%$ de los pacientes con $\mathrm{NH}$, tuvo que ser suspendido por bajo reclutamiento y por el tamaño de la muestra, por lo cual no se pueden sacar conclusiones (28). Un estudio en pacientes con patrón no clasificable del $73 \%$, en el cual el $8 \%$ tenía $\mathrm{NH}$, no cumplió con el objetivo primario en la semana 24 por problemas técnicos y de realización de la espirometría en casa, por lo cual tampoco se pueden obtener conclusiones (29).

\section{Conclusiones}

La neumonitis por hipersensibilidad es otra de las enfermedades pulmonares intersticiales difusas, que debemos sospechar ante la aparición de infiltrados intersticiales en una tomografía de tórax. Es una enfermedad intersticial que tiene etiología y debemos continuar buscando e identificando el antígeno o los antígenos causantes de la enfermedad. Requerimos de una historia completa, un cuestionario pendiente de validar por la región o zona geográfica específica; para cada antígeno, una ayuda tomográfica y patológica para su diagnóstico. Los patrones radiológico e histopatológico de la forma fibrótica y no fibrótica nos permiten una mejor aproximación diagnóstica de la entidad. 
El equipo multidisciplinario debe jugar un papel preponderante en el análisis de los antecedentes, exposiciones, diagnóstico, tratamiento y seguimiento de los pacientes con neumonitis por hipersensibilidad.

Faltan estudios aleatorizados randomizados o consenso de expertos para definir el tratamiento de esta enfermedad en la forma no fibrótica y fibrótica. El retiro de la exposición al antígeno continúa siendo el tratamiento más efectivo de la enfermedad. Están pendientes los estudios específicos para la $\mathrm{NH}$ con esteroides, inmunosupresores y antifibróticos para definir su manejo, pronóstico y sobrevida.

\section{Referencias}

1. Costabel U, Miyazaki Y, Pardo A, Koschel D, Bonella F, Spagnolo P, Guzmán J, Ryerson CJ, Selman M. Hypersensitivity pneumonitis. Nat Rev Dis Primers. 2020;6(1):65. doi: 10.1038/ s41572-020-0191-z

2. Raghu G, Jardin M, Ryerson C, Myers J, et al. Diagnosis Hypersensitivity Pneumonitis in Adults. An official ATS/JRS/ALAT Clinical Practice Guideline. Am J Respir Crit Care Med 2020;202:e36-e69. doi: $10.1164 / \mathrm{rccm} .202005-2032 \mathrm{ST}$

3. Richerson HB, Bernstein IL, Fink JN, Hunninghake GW, Novey HS, et al. Guidelines for the clinical evaluation of hypersensitivity pneumonitis: report of the Subcommittee on Hypersensitivity Pneumonitis. J Allergy Clin Immunol. 1989;84:839-844. doi: 10.1016/00916749(89)90349-7

4. Vasakova M, Morell F, Walsh S, Leslie K, Raghu G. Hypersensitivity pneumonit is: perspectives in diagnosis and management. Am J Respir Crit Care Med. 2017;196:680689. doi: 10.1164/ rccm.201611-2201PP

5. Coultas DB, Zumwalt RE, Black WC, Sobonya RE. The epidemiology of interstitiallung diseases. Am J Respir Crit Care Med 1994;150:967-972. doi: 10.1164/ajrccm.150.4.7921471

6. Fernández Pérez ER, Kong AM, Raimundo K, Koelsch TL, Cole AL. Epidemiology of hypersensitivity pneumonitis among an insured population in the United States: a claims-based cohortanalysis. Ann Am Thorac Soc. 2018;15:460469. doi: 10.1513/AnnalsATS.201704-288OC

7. Buchvald F, Petersen BL, Damgaard K, Deterding $\mathrm{R}$, Langston $\mathrm{C}$, et al. Frequency, treatment, and functional outcome in children with hypersensitivity pneumonitis. PediatrPulmonol. 2011;46:1098. doi: 10.1002/ppul.21479

8. Fernández Pérez ER, Swigris JJ, Forssen AV, Tourin O, Solomon JJ, Huie TJ, et al. Identifying an inciting antigen is associated with improved survival in patients with chronic hypersensitivity pneumonitis. Chest 2013;144:1644-1651. doi: $10.1378 /$ chest.12-2685

9. Wang P, Jones KD, Urisman A, Elicker BM, Urbania T, Johannson $\mathrm{K}$, et al. Pathologic findings and prognosis in a large prospective cohort of chronic hypersensitivity pneumonitis. Chest. 2017;152:502-509. doi: 10.1016/j. chest.2017.02.011

10. Vasakova M, Selman M, Morell F, Sterclova M, Molina-Molina M, Raghu G. Hypersensitivity pneumonitis: current concepts of pathogenesis and potential targets for treatment. Am J Respir Crit.1Care Med. 2019;200:301-308. doi: 10.1164/ rccm.201903-0541PP

11. CottinV, Wollin L, FischerA, Quaresma M, et al. Fibrosing Interstitial lung diseases: Knowns and Unknows. Eur Respir Rev. 2019:28. doi: 10.1183/16000617.0100-2018

12. Morisset J, Johannson KA, Jones KD, Wolters PJ, Collard HR, et al. Delphi Collaborators. Identification of diagnostic criteria for chronic hypersensitivity pneumonitis: an international modified Delphi survey. Am J Respir Crit Care Med 2018;197:1036-1044. doi: 10.1164/ rccm.201710-1986OC

13. Selman M, Teran L, Mendoza A, Camarena A, Martónez-Cordero Lezama $M$, et al. Increase of HLA-DR7 in pigeon breeder's lung in a Mexican population. Clin Immunol Immunopathol.1987;44:63. doi: 10.1016/00901229(87)90052-3

14. Ley B, Newton CA, Arnould I, Elicker BM, Henry TS, Vittinghoff E, et al. The MUC5B promoter polymorphism and telomere length in patients with chronic hypersensitivity pneumonitis: an observational cohort-control study. Lancet Respir Med. 2017;5:639-647. doi: 10.1016/S2213-2600(17)30216-3

15. Raghu G, Remy-Jardin M, Myers JL, Richeldi L, Ryerson CJ, et al. American Thoracic Society; European Respiratory Society; Japanese Respiratory Society; Latin American Thoracic Society. Diagnosis of idiopathic pulmonary fibrosis: an official ATS/ERS/JRS/ALAT clinical practice guideline. Am J Respir Crit 
Care Med. 2018;198:e44-e68. doi: 10.1164/ rccm.201807-1255ST

16. Churg A, Muller NL, Flint J, Wright JL. Chronic hypersensitivitypneumonitis. Am J Surg Pathol. 2006;30:201-208. doi: 10.1097/01. pas.0000184806.38037.3c

17. Salisbury ML, Gross BH, Chughtai A, Sayyouh M, Kazerooni EA,Bartholmai BJ, et al. Development and validation of a radiological diagnosis model for hypersensitivity pneumonitis. Eur Respir J. 2018;52:1800443. doi: 10.1183/13993003.004432018

18. Elicker BM, Jones KD, Henry TS, Collard HR. Multidisciplinary approach to hypersensitivity pneumonitis. J Thorac Imaging. 2016;31:92-103. doi: 10.1097/RTI.0000000000000186

19. Salisbury ML, Myers JL, Belloli EA, Kazerooni EA, Martinez FJ, Flaherty KR. Diagnosis and treatment of fibrotic hypersensitivity pneumonia: where we stand and where we need to go. Am J Respir Crit Care Med. 2017;196:690-699. doi: 10.1164/rccm.201608-1675PP

20. Meyer KC, Raghu G, Baughman RP, Brown KK, Costabel U, du Bois RM, et al.; American Thoracic Society Committee on BAL in Interstitial Lung Disease. An official American Thoracic Societyclinical practice guideline: the clinical utility of bronchoalveolar lavage cellular analysis in interstitial lung disease. Am J Respir Crit Care Med 2012;185:1004-1014. doi: 10.1164/rccm.201202-0320ST

21. Pajares V, Puzo C, Castillo D, Lerma E, Montero MA, Ramos-Barbon D, et al. Diagnostic yield of transbronchial cryobiopsy in interstitial lung disease: a randomized trial. Respirology. 2014;19:900-906. doi: 10.1111/resp.12322

22. Johannson K, Marcoux V, RonsleyP, Ryerson C. Diagnostic yield and complications of transbronchial lung cryobiopsy for intersticial lung disease. A systematic review and Metaanalysis. Annals ATS. 2016;13:1828-1838. doi:

\subsection{3/AnnalsATS.201606-461SR}

23. Morell F, Reyes L, Domenech G, De Gracia J, Majo' J, Ferrer J. Diagnoses and diagnostic procedures in 500 consecutive patients with clinical suspicion of interstitial lung disease [in Spanish]. Arch Bronconeumol. 2008;44:185191. doi: 10.1016/S1579-2129(09)60014-8

24. Salisbury ML, Gu T, Murray S, Gross BH, Chughtai A, Sayyouh M, et al. Hypersensitivity pneumonitis: radiologic phenotype associated with distinct survival time and pulmonary function trajectory Chest. 2018;155:699-711. doi: 10.1016/j.chest.2018.08.1076

25. Morisset J, Johannson KA, Vittinghoff E, Aravena C, Elicker BM, Jones KD, Fell CD, Manganas H, Dube' BP, Wolters PJ, et al. Use of mycophenolate mofetil or azathioprine for the management of chronic hypersensitivity pneumonitis. Chest. 2017; 151:619-625. doi: 10.1016/j.chest.2016.10.029

26. Flaherty K, Wells A, Cottin V, Devaraj A, Walsh S.et al: Nintedanib in progressive fibrosing Interstitial lung diseases. N Engl J Med. 2019; 381:1718-1727. doi: 10.1056/NEJMoa1908681

27. Fernández E,TravisW,Lynch D,Brown K,Johansson K, et al. Diagnosis and Evaluation of Hypersensitivty Pneumonitis.Chest. 2021;e98156. doi: 10.1016/j.chest.2021.03.066

28. BehrJ,Prasse A,KreuterM,Johannes J,Rabe Ketal, Pirfenidone in patients with progressive fibrotic interstitiallung diseases other than idiopathic pulmonary fibrosis(RELIEF): a double-blind, randomised, placebo-controlled,phase $2 \mathrm{~b}$ trial. The Lancet Respir.2021;9:476-486. doi: 10.1016/ S2213-2600(20)30554-3

29. Maher T,CorteT,Fischer A,Kreuter M,Lederer J. et al, Pirfenidone in patients with unclassifiable progressive fibrosing interstitial lung disease: a double-blind, randomised, placebo-controlled, phase 2 trial. The Lancet Respir.2020, 8:147-157. doi: 10.1016/S2213-2600(19)30341-8 